\title{
Leveraging RNA-Seq to Characterize Resistance to Brown Stem Rot and the Rbs3 Locus in Soybean
}

\author{
Chantal E. McCabe, ${ }^{1}$ Silvia R. Cianzio, ${ }^{2}$ Jamie A. O’Rourke, ${ }^{1}$ and Michelle A. Graham ${ }^{1, \dagger}$ \\ ${ }^{1}$ United States Department of Agriculture-Agricultural Research Service Corn Insects and Crop Genetics Research Unit, Ames, \\ IA 50011-1010, U.S.A. and Department of Agronomy, lowa State University, Ames; and ${ }^{2}$ Department of Agronomy, lowa State \\ University
}

Accepted 27 April 2018.

\begin{abstract}
Brown stem rot, caused by the fungus Phialophora gregata, reduces soybean yield by up to $38 \%$. Although three dominant resistance loci have been identified ( $R b s 1$ to $R b s 3$ ), the gene networks responsible for pathogen recognition and defense remain unknown. Further, identification and characterization of resistant and susceptible germplasm remains difficult. We conducted RNA-Seq of infected and mock-infected leaf, stem, and root tissues of a resistant (PI 437970, Rbs3) and susceptible (Corsoy 79) genotype. Combining historical mapping data with genotype expression differences allowed us to identify a cluster of receptor-like proteins that are candidates for the $R b s 3$ resistance gene. Reads mapping to the $R b s 3$ locus were used to identify potential novel single-nucleotide polymorphisms within candidate genes that could improve phenotyping and breeding efficiency. Comparing responses to infection revealed little overlap in differential gene expression between genotypes or tissues. Gene networks associated with defense, DNA replication, and iron homeostasis are hallmarks of resistance to $P$. gregata. This novel research demonstrates the utility of combining contrasting genotypes, gene expression, and classical genetic studies to characterize complex disease resistance loci.
\end{abstract}

In 2015, the United States produced 106 billion $\mathrm{kg}$ (3.93 billion bushels) of soybean (Glycine max) valued at US\$34.5 billion (SoyStats 2015). High yields are critical for soybean profitability; however, pests and pathogens drastically reduce yield. Brown stem rot (BSR) (Koenning and Wrather 2015), caused by the fungus Phialophora gregata f. sp. sojae (Allington and Chamberlain 1948) (syn. Cadophora gregata) (Harrington and McNew 2003) has been reported to cause yield losses of up to $38 \%$

The USDA is an equal opportunity provider and employer. Mention of trade names or commercial products in this article is solely for the purpose of providing specific information and does not imply recommendation or endorsement by the USDA.

${ }^{\dagger}$ Corresponding author: Michelle A. Graham;

E-mail: Michelle.Graham@ars.usda.gov

Funding: This research was financed by the United States Department of Agriculture (USDA) Agricultural Research Service CRIS Project 503021220-005-00D, the Iowa Soybean Association, and the Iowa State University Department of Agronomy.

*The $e$-Xtra logo stands for "electronic extra" and indicates that 4 supplementary figures and 13 supplementary tables are published online.

This article is in the public domain and not copyrightable. It may be freely reprinted with customary crediting of the source. The American Phytopathological Society, 2018.
(Bachman and Nickell 2000). Two types (A and B) of $P$. gregata have been identified. Both cause internal stem browning of the pith and vascular tissue. Only type A causes foliar symptoms such as leaf necrosis and chlorosis (Gray 1972; Harrington et al. 2003). Typically, screening for BSR resistance is conducted in growth chambers and greenhouses 5 weeks after inoculation. Screening methods are time consuming, requiring both foliar and internal stem severity measurements (McCabe et al. 2016). Although $P$. gregata is an economically important pathogen, few genomic tools have been used to characterize resistance.

Genetic resistance to BSR is the most effective tool to reduce yield loss. To facilitate marker-assisted breeding, several groups have mapped BSR resistance $(R)$ genes. Lewers et al. (1999) used restriction fragment length polymorphism and resistance gene analog markers to map Rbs3. Klos et al. (2000) developed simple sequence repeat (SSR) markers for the region corresponding to $R b s 3$ identified by Lewers et al. (1999). Of the nine markers developed, six correctly distinguished between resistant and susceptible germplasm in a panel of 49 lines with greater than $95 \%$ efficiency. Bachman et al. (2001) used SSR markers to map Rbsl and $R b s 2$. All three studies mapped $R b s 1, R b s 2$, and $R b s 3$ to overlapping regions on soybean chromosome 16 (Bachman et al. 2001; Klos et al. 2000; Lewers et al. 1999), though the mapping intervals were large, ranging from 5 to 33 centimorgans. Additionally, novel sources of BSR resistance also map to the $R b s$ loci on chromosome 16 (Perez et al. 2010).

Previous allelism (Hanson et al. 1988; Sebastian and Nickell 1985; Willmont and Nickell 1989) and mapping studies suggest that $R b s 1, R b s 2$, and $R b s 3$ are distinct loci. However, recently, Rincker et al. (2016a) mapped the three known $R b s$ genes to a 340-kb interval on chromosome 16. A subsequent genomewide association study narrowed this region to a 41-kb interval (Rincker et al. 2016b). Based on these findings, Rincker et al. (2016a) suggested that all BSR resistance is conferred by a single gene located within the 41-kb interval.

These studies demonstrate the difficulties and confusion in genetic mapping of complex disease resistance traits and demonstrate a need to better understand disease resistance mechanisms. Development of molecular markers tightly linked to resistance or downstream defense pathways will allow screening to occur prior to BSR symptom development and will improve the efficiency of marker-assisted selection, increasing genetic gain. Examining gene expression differences between genotypes and in response to $P$. gregata infection will aid in identification of genes, networks, and markers contributing to resistance and defense.

The objectives of this study were to use RNA-Seq to characterize candidate resistance genes in the Rbs3 locus and identify genes and networks contributing to defense. To achieve 
these objectives, we used gene expression analyses (RNA-Seq) of leaf, stem, and root tissues in a resistant (PI 437970, containing $R b s 3$ ) and susceptible (Corsoy 79) soybean line, 1 week after $P$. gregata or mock inoculation. We leveraged historical mapping data with RNA-Seq to characterize genotype expression differences at the $R b s 3$ locus. This analysis revealed that a distinct cluster of defense-related receptor-like proteins (RLP) is associated with the Rbs3 locus, and clusters of RLP are also likely associated with resistance at the $R b s 1$ and $R b s 2$ loci. Reads mapping to the Rbs3 locus were used to identify potential single-nucleotide polymorphisms (SNP) that can be used to improve breeding efficiency and fine-map resistance. Comparing responses to $P$. gregata infection in resistant and susceptible germplasm revealed the signatures of defense. These results demonstrate that RNA-Seq expression analyses coupled with mapped resistance loci can be used to effectively identify candidate downstream defense gene networks and to characterize resistance loci, which continues to be difficult in crop species. This approach can be broadly applied to a variety of soybean pathogens and could be used to study disease resistance across a broad range of plant species.

\section{RESULTS}

\section{Verification of BSR phenotype.}

Because expression studies characterizing responses to $P$. gregata infection have not been previously conducted, leaf, stem, and root tissue for RNA-Seq were collected from resistant and susceptible seedlings $1,2,3$, and 4 weeks after infection or mock treatment. Due to the fast advance of BSR symptoms in susceptible infected plants, RNA from samples collected at later time points $(2,3$, and 4 weeks postinoculation) was degraded and not suitable for RNA-Seq analysis. Therefore, only tissue from 1 week postinoculation was used in this study, allowing us to compare between resistant and susceptible genotypes.

Because tissues were collected prior to disease symptom development, additional seedlings of each genotype-treatment combination were allowed to grow for 5 weeks to confirm expected BSR symptom development using three standard screening methods (McCabe et al. 2016). Appropriate BSR phenotypes were observed for each genotype by treatment combination using all three screening methods (Supplementary Table S1.)

\section{Analysis of differentially expressed genes.}

RNA-Seq libraries were sequenced and mapped to the soybean reference genome, Wm82.a2.v1 (Schmutz et al. 2010). In total, 30 samples were analyzed, with 10 samples from each tissue. In leaves, two resistant-infected, two resistant-mock, three susceptible-infected, and three susceptible-mock samples were included in the analysis. In stems, two resistant-infected, two resistant-mock, three susceptible-infected, and three susceptiblemock samples were analyzed. In roots, two resistant-infected, three resistant-mock, three susceptible-infected, and two susceptible-mock samples were analyzed. The 30 samples analyzed contained a total of 427,668,168 100-bp single end reads. These sequences are available from the National Center for Biotechnology Sequence Read Archive, BioProject Accession SRP092482. Statistically significant (false discovery rate $[F D R]<0.001)$ differentially expressed genes (DEG) responding to genotype, treatment, treatment within a genotype, and treatment-genotype interaction were identified for each tissue. Log fold-change, log counts per million, and FDR for all statistical comparisons are provided in the supplementary materials.

To identify overlapping responses, DEG responding to genotype and $P$. gregata infection were compared between genotypes and between tissues (Table 1). Although thousands of DEG were identified in these analyses, fewer than 200 genes were common between genotypes in response to infection and less than 40 were common between tissues of a given genotype, highlighting the dynamic responses to $P$. gregata infection.

\section{Characterization of the $\boldsymbol{R} \boldsymbol{b} \boldsymbol{s} \mathbf{3}$ locus by RNA-seq.}

We identified 6,938 significant DEG (FDR $<0.001$ ) between resistant and susceptible genotypes, regardless of pathogen infection (Supplementary Table S2). Because lines used in this study were unrelated, we expected that most of these genotype

Table 1. Number of significant (false discovery rate <0.001) differentially expressed genes (DEG) responding to genotype $(\mathrm{G})$, Phialophora gregata treatment (T), genotype-treatment interaction $(\mathrm{G} \times \mathrm{T})$, and genotype-specific response to P. gregata infection (Inf) in leaf, stem, and root tissue ${ }^{\mathrm{a}}$

\begin{tabular}{|c|c|c|c|c|c|c|c|}
\hline \multirow[b]{3}{*}{ Measured } & \multicolumn{7}{|c|}{ DEG $^{\mathbf{b}}$} \\
\hline & \multirow[b]{2}{*}{ All genotypes } & \multirow[b]{2}{*}{$\operatorname{Res} \boldsymbol{R} b s \mathbf{3}$} & \multirow[b]{2}{*}{ Sus } & \multicolumn{4}{|c|}{ Number in common } \\
\hline & & & & Leaves, stems & Leaves, roots & Stems, roots & Res, Sus \\
\hline \multicolumn{8}{|c|}{$\mathrm{G}$ effect (Rbs3/Sus) } \\
\hline Leaves & $1,901+, 2,230-$ & $\ldots$ & $\ldots$ & $\ldots$ & $\ldots$ & $\ldots$ & $\ldots$ \\
\hline Stems & $1,017+, 1,573-$ & $\ldots$ & $\ldots$ & $\ldots$ & $\ldots$ & $\ldots$ & $\ldots$ \\
\hline Roots & $1,228+, 866-$ & $\ldots$ & $\ldots$ & $\ldots$ & $\ldots$ & $\ldots$ & $\ldots$ \\
\hline \multicolumn{8}{|c|}{$\mathrm{T}$ effect (Inf/Mock) } \\
\hline Leaves & $160+, 135-$ & $976+, 1,516-$ & $16+, 73-$ & $\ldots$ & $\ldots$ & $\ldots$ & $\ldots$ \\
\hline Stems & $647+, 266-$ & $462+, 68-$ & $297+, 127-$ & $\ldots$ & $\ldots$ & $\ldots$ & $\ldots$ \\
\hline Roots & $1+, 1-$ & $4+, 12-$ & $55+, 108-$ & $\ldots$ & $\ldots$ & $\ldots$ & $\ldots$ \\
\hline \multicolumn{8}{|c|}{$\mathrm{G} \times \mathrm{T}(\operatorname{Rbs} 3[\mathrm{Inf} / \mathrm{Mock}] / \mathrm{Sus}[\mathrm{Inf} / \mathrm{Mock}])$} \\
\hline Leaves & 974 & $\ldots$ & $\ldots$ & $\ldots$ & $\ldots$ & $\ldots$ & $\ldots$ \\
\hline Stems & 37 & $\ldots$ & $\ldots$ & $\ldots$ & $\ldots$ & $\ldots$ & $\ldots$ \\
\hline Roots & 44 & $\ldots$ & $\ldots$ & $\ldots$ & $\ldots$ & $\ldots$ & $\ldots$ \\
\hline \multicolumn{8}{|c|}{ Between tissues ${ }^{\mathrm{c}}$} \\
\hline Resistant & $\ldots$ & $\ldots$ & $\ldots$ & 36 & 2 & 1 & $\ldots$ \\
\hline Susceptible & $\ldots$ & $\ldots$ & $\ldots$ & 21 & 8 & 16 & $\ldots$ \\
\hline \multicolumn{8}{|c|}{ Between responses $^{\mathrm{d}}$} \\
\hline Leaves & $\ldots$ & $\ldots$ & $\ldots$ & $\ldots$ & $\ldots$ & $\ldots$ & 25 \\
\hline Stems & $\ldots$ & $\ldots$ & $\ldots$ & $\ldots$ & $\ldots$ & $\ldots$ & 173 \\
\hline Roots & $\ldots$ & $\ldots$ & $\ldots$ & $\ldots$ & $\ldots$ & $\ldots$ & 0 \\
\hline
\end{tabular}


expression differences would be unrelated to BSR resistance. However, because Rbs3 had been mapped by Lewers et al. (1999) (Gm16, 33.3 to $33.8 \mathrm{Mbp}$ ), we could examine genotype expression differences between our resistant and susceptible line at the Rbs 3 locus (Supplementary Table S3). Of the 37 genes in the mapped $R b s 3$ region, 21 genes were statistically differentially expressed between genotypes. Of these, 18 were expressed at higher levels in the resistant genotype (fold change [FC] ratios between 1.74 and 271). Strongest differential expression occurred in root tissue of 12 genes with homology to RLP. RLP play an important role in both the perception of and resistance to pathogens that grow extracellularly (Kruijt et al. 2005). The Rbs 3 RLP are homologous to apple HcrVf genes, which provide resistance to apple scab; the tomato $C f$, Ve, and LeEIX genes, which provide resistance to Cladosporium fulvum, Verticillium wilt, and Trichoderma viride, respectively; and the Arabidopsis gene RPP27, which provides resistance to Peronospora parasitica (Kruijt et al. (2005)). We also identified four UDP-glucose transferases (UGT) in this region that were differentially expressed in leaves. UGT also play roles in plant defense. In Arabidopsis, AtUGT73B5 and AtUGT73B3 are involved in the hypersensitive response (Simon et al. 2014). AtUGT73C5 conjugates and detoxifies the fungal mycotoxin produced by Fusarium spp. (Poppenberger et al. 2003). Of the four UGT identified, only two were expressed more in the resistant genotype (Glyma.16G17550 [FC = 3.24] and Glyma. $16 \mathrm{G} 175600[\mathrm{FC}=1.73])$.

We also identified DEG responding to Phialophora gregata infection that were located within the Rbs3 locus (Supplementary Table S4). Only eight genes were statistically differentially expressed in response to infection. Of these, only three had roles associated with defense and were induced by infection in the resistant parent. These included a chitinase (Glyma.16G173000 $[\mathrm{FC}=72.7]$, resistant-infected/resistant mock) and two UGT (Glyma.16G175300 [FC = 4.38] and Glyma.16G176000 [FC = 2.78]), all induced in stems of the resistant parent.

\section{Characterization of candidate resistance genes in all $R b s$ loci.}

Given that RLP were associated with $R b s 3$ resistance, we wanted to determine whether RLP were associated with the other Rbs loci on chromosome 16. Using historical mapping data (Bachman et al. 2001; Klos et al. 2000; Lewers et al. 1999; Rincker et al. 2016a,b), we examined the region between markers Satt215 (Gm16, 28.9 Mbp) and Satt431 (Gm16, 36.2 Mbp), spanning all Rbs loci on chromosome 16. Of the 687 predicted genes in this region, 127 had homology to potential $R$ genes, including 107 RLP, two receptor-like kinases (RLK), and 18 nucleotide-binding site leucine-rich repeats (NBSLRR). We used InterProScan (Jones et al. 2014) to characterize these genes (Supplementary Table S5). The RLP and RLK contained a signal peptide, a range of LRR motifs (PF08623, SM00369, PF13855, PR00019, SM00365, and PS51450), a transmembrane domain, and a short cytoplasmic domain (Fig. 1). The RLK contained an additional protein kinase domain (PF00069). The NBS-LRR contained a Toll/interleukin1 receptor domain, a nucleotide-binding ARC domain (NB-ARC) (SM00255), and an LRR domain (IPR032675). Three of the NBSLRR lacked one or more of these domains (Glyma.16G159500, Glyma.16G159700, and Glyma.16G159800). We subgrouped the RLP by LRR domain (LRR-A or LRR-B). We classified 80 RLP with LRR-A domains, containing all of the InterProScanpredicted LRR domains except PS51450, with an average of nine LRR repeats. We classified 27 RLP as LRR-B, always containing PS51450 among other LRR domains, with an average of 19 LRR repeats. Of the 107 predicted RLP in the Rbs loci, only 65 were full length and contained LRR motifs, a transmembrane domain, and a short cytoplasmic domain.
Unlike NBS-LRR, which are specific to resistance, RLP can function in resistance or development. Fritz-Laylin et al. (2005), demonstrated that resistance-associated RLP had two conserved cysteine pairs within a conserved B-domain, although developmentassociated RLP contained a single pair. Aligning the B-domain of the 65 full-length RLP revealed two pairs of conserved cysteines, suggesting the $R b s$ RLP function in defense. The alignment also clearly divided the RLP into five distinct classes, B1 to B5 (Supplementary Fig. S1), containing 13, 5, 9, 22, and 16 RLP, respectively. The RLP contain many hallmarks of resistance genes, including conserved resistance motifs, clustering, and numerous deletions, which are evidence of unequal recombination.

By combining all $127 R$ genes, RLP B-domain classification, physical location, expression, and historical $R b s$ mapping data, we identified distinct RLP clusters within the $R b s$ loci (Fig. 2). The entire region depicted in Figure 2 corresponds to the $R b s 1$ locus, which includes $R b s 2$ and $R b s 3$. The region unique to Rbs 1 contains 26 RLP, 17 NBS-LRR, and two RLK. Of these, three RLP and three NBS-LRR were significantly differentially expressed in resistant roots where $P$. gregata infection naturally occurs. The $R b s 2$ locus corresponded to 68 RLP and an NBSLRR. Only a single RLP was significantly differentially expressed in resistant roots. The Rbs 3 locus contained 13 RLP (8 full length in the Williams82 reference) and was specifically associated with RLP class B3. Twelve of these were significantly differentially expressed more in roots of the $R b s 3$ resistant genotype and are likely candidate genes for $R b s 3$ (Glyma.16G173900, Glyma.16G174000, Glyma.16G174100, Glyma.16G174400, Glyma.16G174500, Glyma.16G174600, Glyma.16G174700, Glyma.16G174800, Glyma.16G175000, Glyma.16G175100, Glyma.16G176400, and Glyma.16G176600). Our findings suggest that RLP are responsible for $P$. gregata resistance at the $R b s 3$ locus and are likely associated with resistance at the Rbsl and Rbs2 loci.

\section{De novo assembly of the $R b s 3$ Locus.}

Previous research has shown that disease resistance loci often vary in candidate resistance gene copy number across different genotypes (Anderson et al. 2014; McHale et al. 2012). Mapping transcripts to the reference genome may collapse copies of candidate resistance genes. Therefore, in order to identify novel transcript variants not present in the Williams 82 reference genome, we developed a de novo assembly from all reads from all tissues, treatments, and genotypes mapping between 32.72 and 34.25 Mbp on chromosome 16, corresponding to the previously

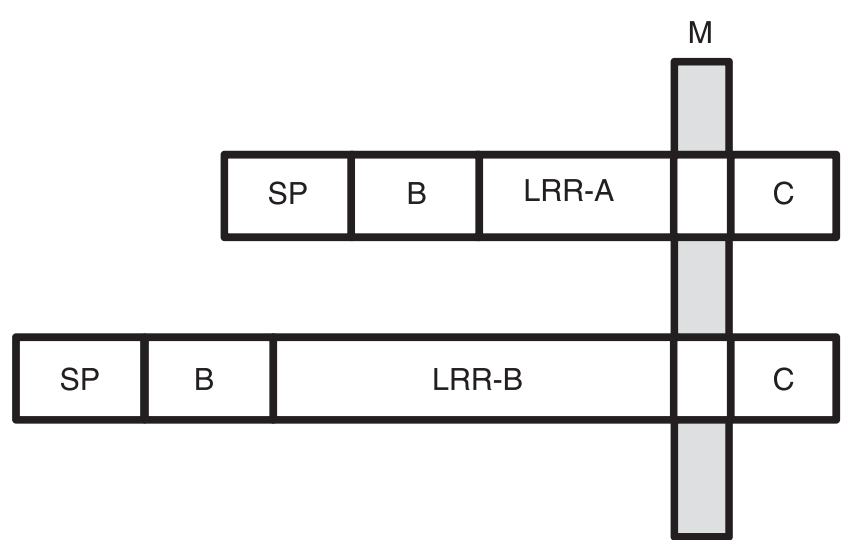

Fig. 1. Receptor-like protein (RLP) domain structure. Five domains make up RLP: signal peptide (SP), B-domain (B), leucine-rich repeat (LRR) domain, transmembrane domain $(\mathrm{M})$, and the cytoplasmic domain $(\mathrm{C})$. Variations exist within the B-domain (B1, B2, B3, B4, and B5) and the LRR domain (LRR-A and LRR-B). 
mapped Rbs3 locus (Lewers et al. 1999) and the fine-mapped $R b s$ locus (Rincker et al. 2016a). This resulted in 508 transcripts representing 289 genes (Supplementary Table S6). Within the de novo assembly, we identified genotype, treatment, and genotype-treatment DEG (FDR < 0.001). We used $\log 2$ counts per million (cpm) data for each sample to create a heatmap of the 53 genes differentially expressed in response to genotype across all tissues (Supplementary Table S7; Supplementary Fig. S2).

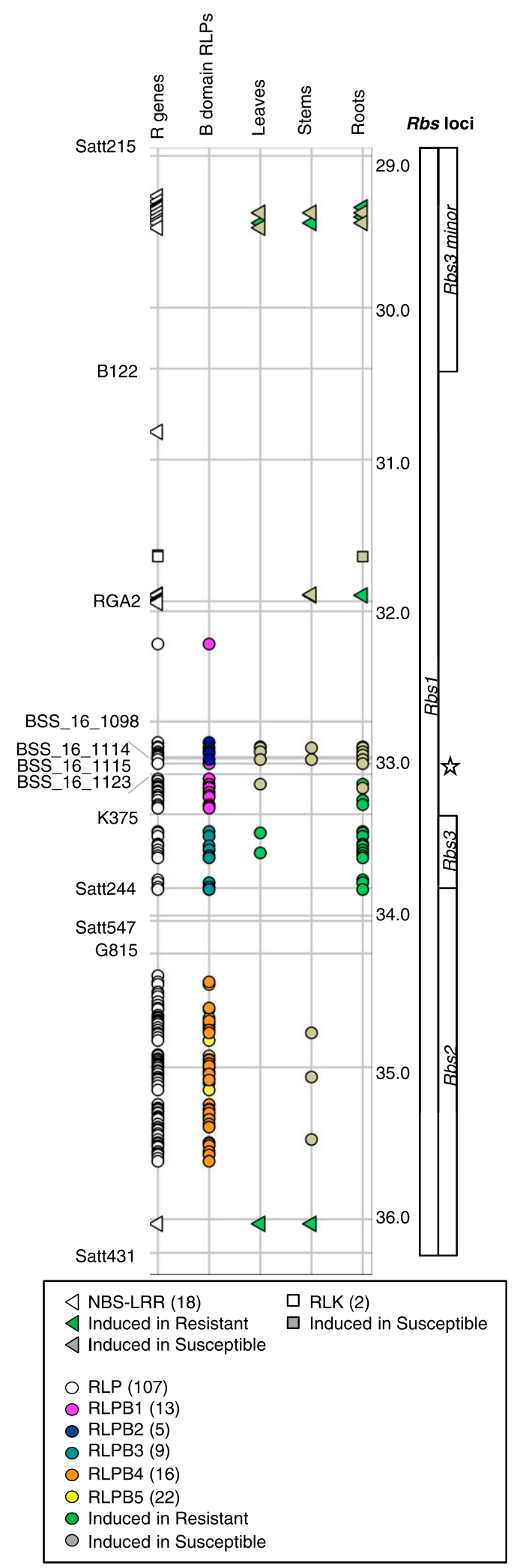

The de novo assembly contained 16 DEG with homology to RLP differentially expressed between genotypes in leaves and roots. Homologs of AtRLP1 (RBS154.c0_g2_i1), AtRLP9 (RBS100.c0_g1_i1), AtRLP12 (RBS173.c1_g2_i1), AtRLP13 (RBS3.c1_g2_i1), AtRLP43 (RBS31.c1_g1_i1), and At4G36180 (RBS173.c1_g4_i1) were expressed more in the resistant genotype. In contrast, homologs of AtRLP24 (RBS114.c1_g1_i1), AtRLP34 (RBS15.c0_g1_i1), AtRLP36 (RBS114.c1_g2_i1), AtRLP38 (RBS121.c0_g1_i1), and AtRLP53 (RBS114.c0_g1_i1) were expressed more in the susceptible genotype. AtRLP33 (RBS173.c1_g1_i1 and RBS158.c0_g1_i1) and At2G34930 (RLP154.c0_g3_i1, RLP157.c0_g1_i1, and RBS100.c2_g2_i1) were significant in both resistant and susceptible genotypes but in different tissues.

Several other DEG also had significantly greater expression in the resistant genotype in our de novo assembly. This included transcripts with greatest homology to AtRCC1 (RBS65.c0_g1_i1) (regulator of chromosome condensation 1), which plays a key role in signal transduction and cell signaling (van Leeuwen et al. 2004). A UGT, AtUGT88A1 (RBS180.c0_g2_i1), was significantly expressed in resistant leaves and roots. As previously mentioned, UGT have been found to play roles in plant stress and defense responses ( $\mathrm{Li}$ et al. 2001). Fourteen transcripts with greater expression in the susceptible genotype were also identified. Only one transcript was identified as being significant in both genotypes, AtVIT (RBS84.c0_g1_i1). Vacuolar iron transporters are nodulin-like genes that function in regulating iron homeostasis in Arabidopsis and uptake iron for storage in the vacuole (Gollhofer et al. 2011).

\section{SNP identification from resistant and susceptible genotypes.}

We were interested in using our RNA-seq data to generate SNP that could be useful to breeders. To allow us to order the SNP across the Rbs3 locus, we merged our original mapping files that mapped reads from the resistant and susceptible genotype to the Williams 82 reference genome. To call SNP, we focused on the expanded $R b s 3$ region (Gm16, 32.72 to 34.25 Mbp). In total, 534 potential SNP with a quality score greater than 25 were identified (Supplementary Table S8). Of these, 502 mapped within genic regions while the remaining 32 mapped within untranslated portions of the gene. In total, SNP were mapped to 66 unique genes. Of the 12 RLP expressed more in resistant roots, we were able to identify potential novel SNP for 5 (Glyma.16G174600, Glyma.16G174700, Glyma. 16G174800, Glyma.16G176400, and Glyma.16G176600). These SNP will be further explored for marker development. SNP identified 1 week after infection will improve breeding efficiency by allowing earlier screening, prior to the onset of BSR symptoms.

Fig. 2. The Rbs3 locus is associated with a class B3 receptor-like protein (RLP). Rbs1, Rbs2, and Rbs3 have been mapped between Satt215 and Satt431 on chromosome 16 (Bachman et al. 2001; Klos et al. 2000; Lewers et al. 1999). In this region, we identified and annotated 107 RLP, 2 receptorlike kinase (RLK), and 18 nucleotide-binding site leucine-rich repeat (NBS-LRR) genes in Williams 82. The B-domains of 65 full-length RLP were aligned and divided into five subgroups (B1 to B5). Although few resistance $(R)$ genes responded to Phialophora gregata infection, several were significantly differentially expressed between resistant and susceptible genotypes. Using RNA-Seq data, $R$ genes significantly differentially expressed between genotypes were identified in leaves, stems, and roots. Genetic markers are indicated to the left of the panel, physical distance is indicated to the right. Historical mapping locations of individual Rbs loci are indicated by vertical boxes. The $R b s$ region identified by Rincker et al. (2016a) is indicated with a star. R was used to visualize the different data types in the $R b s$ region. 


\section{Characterization of genes responding}

\section{to $P$. gregata infection.}

Our analyses thus far have focused on the previously identified $R b s$ loci. However, we were also interested in identifying genes that respond to $P$. gregata infection. To analyze expression trends due to $P$. gregata treatment, we combined the treatment DEG identified in roots, stems and leaves, identifying 3,788 unique treatment DEG (Table 1; Supplementary Table S9). Hierarchical clustering (R CoreTeam 2014) was used to cluster the DEG by expression pattern across all tissues and genotypes (Fig. 3A). Of the 3,788 unique DEG, 181 (5\%) are from roots, 1,867 (49\%) are from stems, and 2,876 (76\%) are from leaves. Therefore, the leaves have largely determined the clustering pattern. In roots, DEG had similar expression patterns across all treatments. In stems, we observed slight differences between genotypes and treatments. In leaves, we observed a distinct expression pattern in the resistant genotype infected with $P$. gregata relative to the resistant mock-infected, susceptible mock-infected, and susceptible infected leaves.

Clustering divided the 3,788 treatment DEG into seven treatment $(\mathrm{T})$ clusters with distinct expression patterns across genotypes or treatments (Fig. 3A). Clusters T1 to T7 contained 678, $135,683,308,281,666$, and 1,037 DEG, respectively. To assign functions to these clusters, gene ontology (GO) biological process (Berardini et al. 2004) terms significantly $(P$ value $<0.05)$ overrepresented within a cluster relative to the soybean genome were identified (Fig. 3B; Supplementary Table S10). Treatment clusters $\mathrm{T} 1$ to $\mathrm{T} 7$ contained 7, 4, 5, 0, 1, 20, and 52 significantly overrepresented GO terms, respectively. Significant GO terms were unique to a cluster. This analysis suggests that clusters of differentially expressed genes have unique biological functions.

Clusters T1, T3, T4, and T5 were primarily composed of DEG from leaves and stems of both genotypes (Fig. 3A). T1 and T3 had similar gene expression patterns in leaves but opposite patterns in stems and roots. T4 and T5 had mixed expression in leaves but were mostly induced in stems and repressed in roots. For each of these clusters, a distinct response was observed in resistant-infected leaves. In stems and roots, few expression differences could be detected across samples. Significantly overrepresented $\mathrm{GO}$ terms in $\mathrm{T} 1$ and $\mathrm{T} 3$ included four terms related to hormone signaling and five terms related to defense and stress responses (Fig. 3B). T5 had a single significantly overrepresented GO term (maltose biosynthetic process, GO:0000024). T4 had no significantly overrepresented GO terms.

Clusters T2, T6, and T7 were composed almost entirely of DEG from resistant leaves (Fig. 3A). In T2, DEG had mixed expression in response to infection. In T6, resistant DEG were induced in response to $P$. gregata. In T7, DEG were repressed in response to infection. Little significant differential expression was observed in the susceptible genotype or any other tissue. T2 contained four significantly overrepresented GO terms related to transport (Fig. 3B). T6 contained 20 significantly overrepresented GO terms, including 7 related to photosynthesis and 9 related to metabolism. T7 contained 52 significantly overrepresented GO terms, 33 related to the cell cycle and 15 related to gene silencing. These results confirm the different responses observed between genotypes and tissues and demonstrate the utility of clustering genes by expression pattern to assign putative biological function. The coregulation of defense, photosynthesis, and metabolism in response to pathogen infection has been observed in other soybean-pathogen systems (Bilgin et al. 2010; Bolton 2009; Kangasjärvi et al. 2012).

\section{Transcription factor analyses}

\section{of $P$. gregata treatment response.}

Given the distinct differences in gene expression caused by $P$. gregata infection, we were interested in identifying transcription factors (TF) within our data set that regulate defense gene networks. Within our 3,788 P. gregata treatment DEG, we identified $215 \mathrm{TF}$ (Supplementary Table S11), representing $30 \mathrm{TF}$ families in the SoyDB TF database (Wang et al. 2010). In the resistant response (Fig. 4A), we observed greater TF expression in leaves and stems, with little differential expression in roots. In contrast, the majority of TF expression in the susceptible response was observed in stems (Fig. 4B). This suggests that the defense signal has spread from stems to leaves in the resistant genotype but not in the susceptible. Alternatively, the leaves in the susceptible genotype may be unable to recognize the defense signal from the stems to induce a response. Further, we identified TF families possibly important in the resistant and susceptible responses. The resistant response included WRKY, MYB-HD, Homeodomain/HOMEOBOX, bHLH, and AP2-EREBP TF families. All but the MYB-HD TF family had largely opposing expression patterns in stems and leaves. These same TF families were also identified in the susceptible response; however, expression was limited to the stems.

To associate TF with the clusters of genes they regulate and a biological function, we also analyzed the TF by their original treatment cluster. Clusters T1, T3, T4, and T5 were composed of leaf and stem DEG from both genotypes and clusters T1 and T3 were associated with stress and defense responses. Clusters $\mathrm{T} 1, \mathrm{~T} 3, \mathrm{~T} 4$, and $\mathrm{T} 5$ contained 35, 69, 34, and $26 \mathrm{TF}$, respectively. All four clusters included several TF families associated with abiotic and biotic stress responses, such as the MYB-HD, AP2-EREBP, and WRKY TF families. Two homologs of $A t M Y B 15$ were significantly induced in stems of both genotypes, while three homologs of AtMYB73 and one homolog of $A t M Y B 77$ were significantly repressed in stems and leaves of both genotypes. AtMYB15, AtMYB73, and AtMYB77 regulate general stress responses such as wounding or cold stress (Cheong et al. 2002; Ding et al. 2009; Fowler and Thomashow 2002). Several ethylene response factors, members of the AP2-EREBP TF family, were identified in T1, T3, and T4. Homologs of AtERF1 (Glyma.10G036700, Glyma.10G186800, Glyma.19G163900, and Glyma.20G203700) were induced in stems of both genotypes. However, Glyma.16G163900 was induced 132-fold in stems of the resistant genotype but only 31-fold in the susceptible. AtERF 1 incorporates both ethylene and jasmonate signaling pathways, regulating expression of pathogen response genes (Gutterson and Reuber 2004). Overexpression of AtERF1 enhances defenses against necrotrophic pathogens (Robert-Seilaniantz et al. 2011). Two homologs of AtERF15 were identified, Glyma.03G162700 and Glyma. 13G123100. Both TF were induced in stems of both genotypes; however, Glyma.03G162700 was induced 362-fold in the resistant genotype but only 244 -fold in the susceptible. AtERF 15 acts as a positive regulator of the immune response against the fungus Botrytis cinerea in Arabidopsis (Zhang et al. 2015). Also induced in stems were homologs of AtERF98, which enhances salt tolerance, protecting plants from reactive oxygen species damage (Zhang et al. 2012). WRKY TF AtWRKY4O and AtWRKY51 negatively regulate jasmonic-acid-inducible defense responses (Gao et al. 2011). Homologs of AtWRKY40 (Glyma.04G061400, Glyma.14G102900, and Glyma.17G222500) and AtWRKY51 (Glyma.05G184500 and Glyma.06G147100) were induced in stems of both genotypes (T3 and T4). However, these WRKY TF had very large expression differences between genotypes. For example, Glyma.14G102900 and Glyma.06G147100 were induced 398- and 46-fold, respectively, in the resistant genotype and only 166- and 21-fold in the susceptible.

Clusters T2, T6, and T7 were composed mostly of leaf DEG specific to the resistant response. Clusters T2, T6, and T7 contained 3, 29, and $19 \mathrm{TF}$, respectively.

Many of the TF in T2, T6, and T7 are associated with DNA replication, cell cycle, gene silencing, and iron homeostasis. 


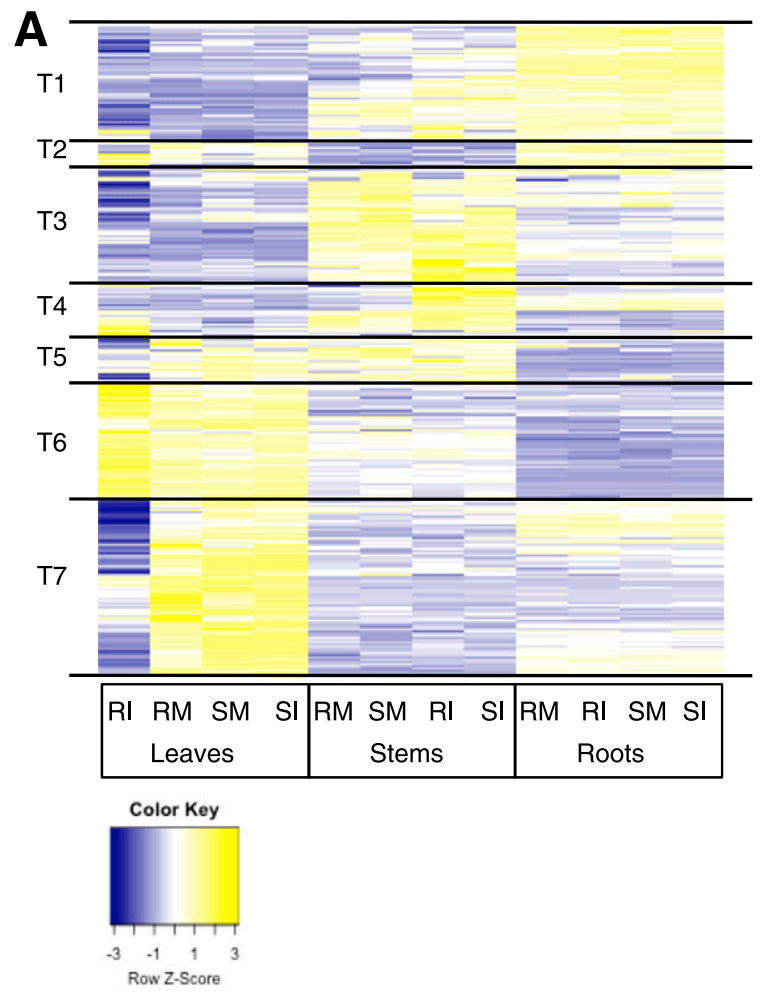

B

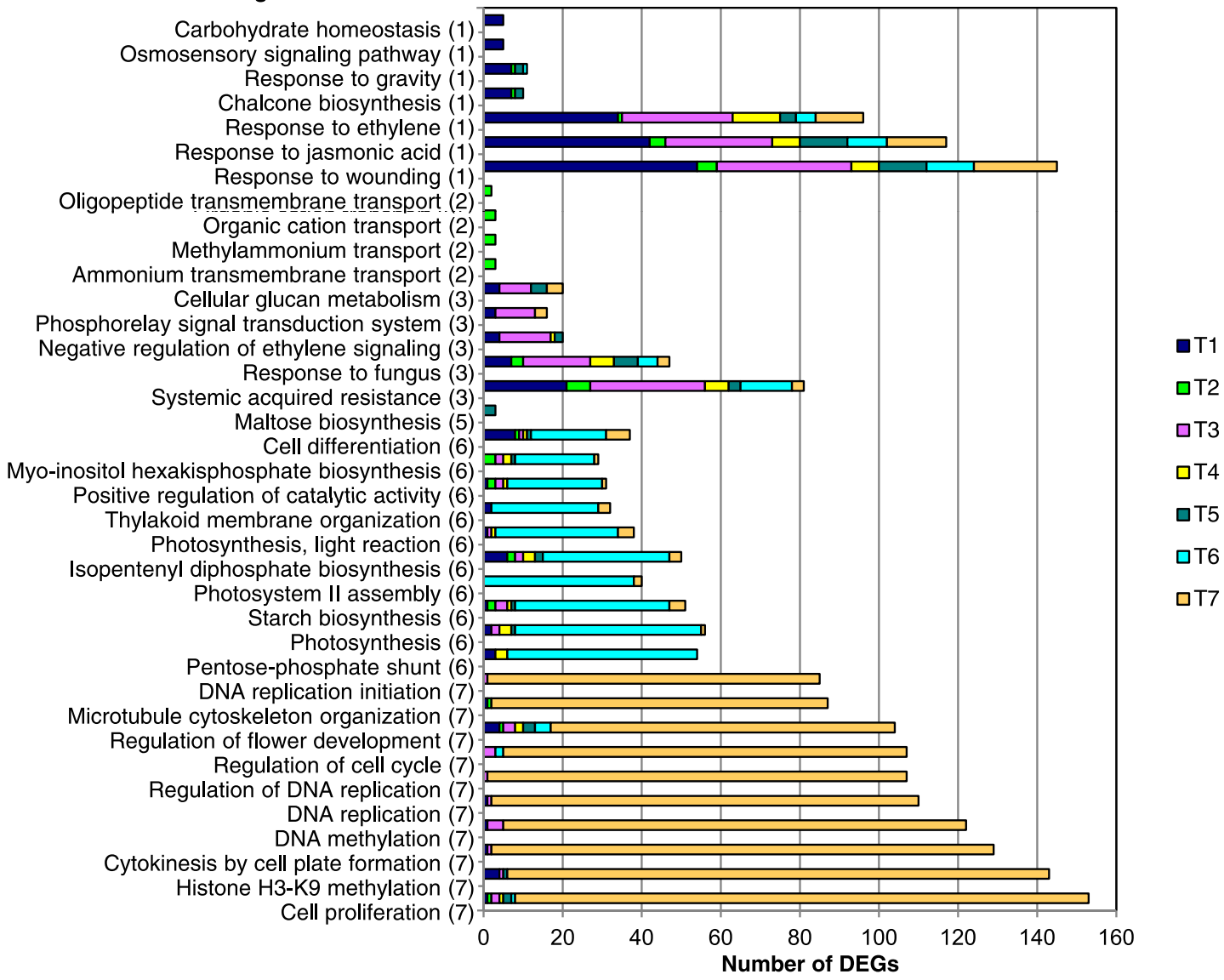

Fig. 3. Hierarchical clustering of Phialophora gregata treatment response. In total, 3,788 significant (false discovery rate $<0.001$ ) unique differentially expressed genes (DEG) responded to treatment; resistant and susceptible response to P. gregata were included. A, Genes were clustered with similar expression patterns using Z-score. As indicated by the colors, a Z-score less than zero indicates lower expression than the mean, and a Z-score greater than zero indicates greater expression than the mean. The columns differentiate the three tissue types and four treatments within each tissue: resistant-mock (RM), resistantinfected (RI), susceptible-mock (SM), and susceptible-infected (SI). Rows group genes by similar expression pattern, forming seven expression clusters (T1 to T7). B, Significantly $(P$ value $<0.05)$ overrepresented gene ontology $(\mathrm{GO})$ terms associated with $P$. gregata treatment clusters. To aid in visualization, clusters were limited to the 10 largest significantly overrepresented GO terms per cluster, if applicable. 
Glyma.07G250700, homolog of AtCHR17, is important in formation of nucleosome distribution patterns in Arabidopsis $(\mathrm{Li}$ et al. 2014). Glyma.01G175300, homologous to AtDDM1, is responsible for transposon and transgene silencing as well as maintaining DNA methylation levels (Gendrel et al. 2002). Glyma.05G189400, homologous to AtTSK, is required for cell cycle progression (Suzuki et al. 2005). Glyma.04G238400 and Glyma.06G125500, homologous to AtSPCH, initiate asymmetric cell divisions in leaves and are essential for stomatal development (Danzer et al. 2015). AtCHR17, AtDDM1, AtTSK, and $A t S P C H$ are all repressed in leaves in T7. Glyma.16G062600 and Glyma.16G06300, induced in leaves in T6, are homologous to

A Resistant Response to $P$. gregata
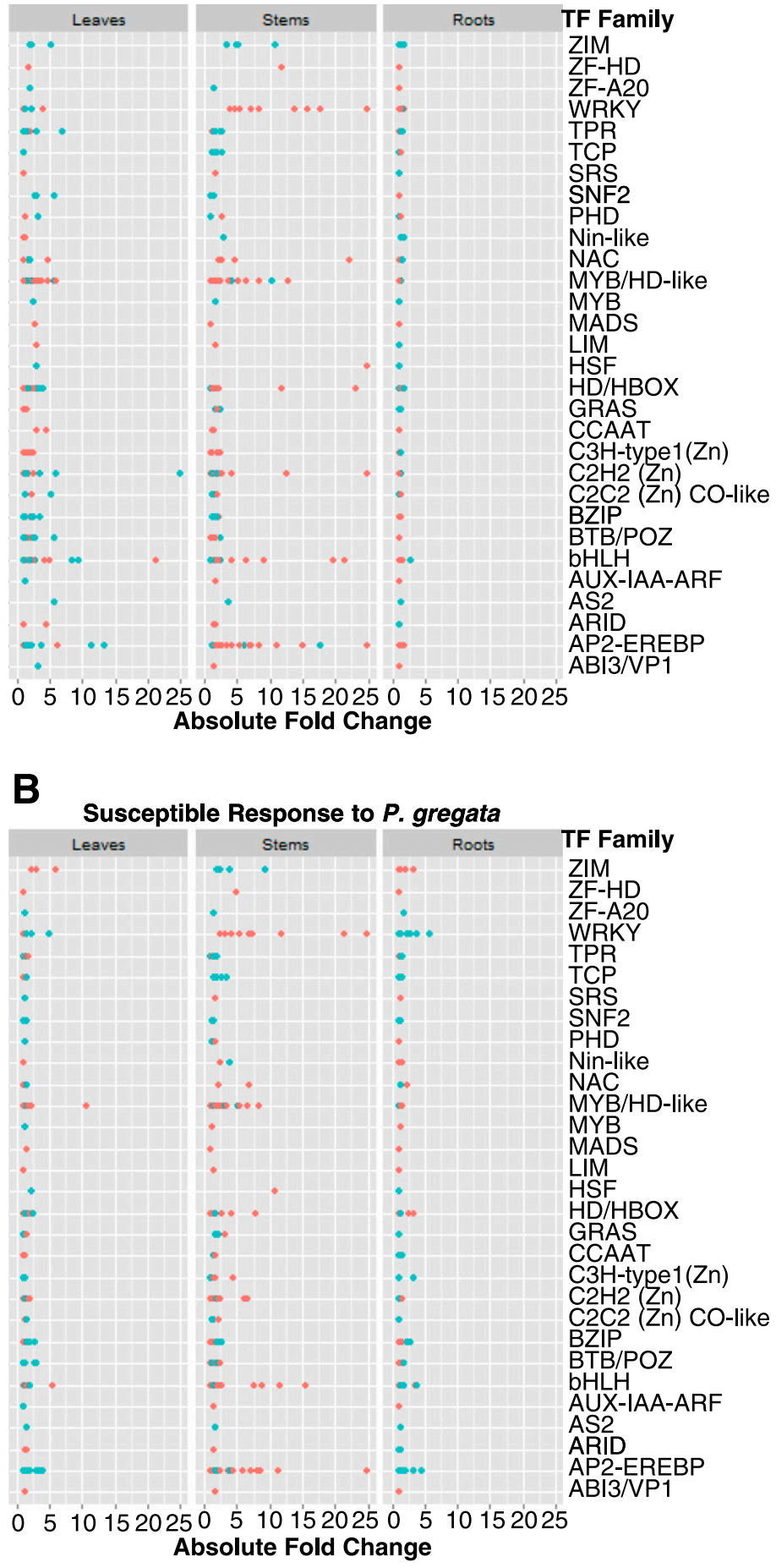

- Induced - Repressed

Fig. 4. Transcription factor (TF) expression in response to Phialophora gregata treatment in resistant and susceptible genotypes. Significantly (false discovery rate $<0.001)$ differentially expressed TF were identified in each of the seven treatment clusters. Expression of differentially expressed TF was observed across genotypes and tissues in both the $\mathbf{A}$, resistant (resistant infected/resistant mock) and $\mathbf{B}$, susceptible (susceptible infected/susceptible mock) responses to P. gregata. Absolute fold change is plotted on the $\mathrm{x}$-axis and TF families are plotted on the y-axis. For visualization purposes, TF with an absolute fold change greater than 25 were plotted as 25 . 
AtbHLH105, a positive regulator of the iron deficiency response in Arabidopsis (Liang et al. 2017).

\section{Hierarchical clustering \\ of $P$. gregata treatment-genotype response.}

In leaves, stems, and roots, we identified 974, 37, and 44 significant DEG, respectively, with a treatment-genotype interaction (FDR < 0.001) (Supplementary Table S12). This analysis identified genes whose treatment response is dependent on genotype. Leaf treatment-genotype (LTG) DEG organized into two clusters, LTG1 and LTG2, with 169 and 805 DEG, respectively. DEG in LTG1 were induced in response to infection in the resistant genotype but repressed in the susceptible genotype. The opposite expression pattern was observed in LTG2 (Supplementary Fig. S3). LTG1 was significantly overrepresented ( $P$ value $<0.05)$, with the GO terms cellular iron ion homeostasis (GO:0006879) and response to zinc ion (GO:0010043) (Supplementary Table S13). LTG2 was significantly overrepresented, with $30 \mathrm{GO}$ terms related to cell cycling, gene silencing, and metabolism.

The stem treatment-genotype (STG) DEG separated into two clusters containing 33 and 4 genes (STG1 and STG2, respectively) (Supplementary Fig. S4). DEG in STG1 were induced in response to infection in the resistant genotype but not the susceptible. DEG in STG2 had the opposite expression pattern. No significantly overrepresented GO terms were identified in either cluster; however, several genes were identified with functions relating to defense. Three homologs of AtMLP423 (Glyma. 17G030200, Glyma.17G030300, and Glyma.17G030400) were significantly induced. AtMLP423 is involved in defense response and is induced by pathogen colonization (Plett et al. 2015).

Root treatment-genotype (RTG) DEG formed two clusters containing 28 and 16 genes (RTG1 and RTG2, respectively). RTG1 was induced in response to infection in the susceptible genotype, while RTG2 was repressed in response to infection in the susceptible genotype. Though weak, the opposite patterns were observed in the resistant genotype. In RTG2, only one significantly overrepresented GO term was identified, lignin metabolic process (GO:0009808).

\section{DISCUSSION}

Understanding genetic mechanisms underlying resistance is crucial in breeding for resistance to $P$. gregata. Phenotyping for BSR is difficult and time consuming, requiring multiple destructive phenotyping measurements 5 to 6 weeks after infection, hindering progress in identifying and characterizing resistance loci necessary for marker-assisted breeding. Traditional genetic studies characterizing resistance have provided conflicting results from single-candidate $R$ genes to complex multigenic traits. To our knowledge, no expression studies have targeted the soybean $-P$. gregata pathosystem. Therefore, we conducted RNA-Seq analyses across multiple tissues and resistant and susceptible genotypes, and in response to $P$. gregata infection. Our findings highlight the utility of RNA-Seq for characterizing $R$ gene loci and downstream defense pathways. Although our analyses were limited to a single time point, our findings suggest that soybean responds much faster to $P$. gregata infection than phenotyping would suggest. This research provides promise for improving the speed and reliability of current phenotyping methods.

Combining our RNA-Seq analyses with historical mapping data has shed light on the complexities of the $R b s$ loci. We identified 107 RLP in the $R b s$ region on chromosome 16. The entire Arabidopsis genome only contains 57 RLP. The RLP contained many hallmarks associated with disease resistance, including conserved resistance motifs (Fritz-Laylin et al. 2005) and clustering within the genome. Clustering allows for rapid evolution of novel pathogen specificities through duplication and gene recombination events but could also cause localized deletions.

Within the Rbs3 locus, few genes responded to $P$. gregata infection; however, many were differentially expressed between genotypes in roots. This suggests that $P$. gregata resistance is not inducible; instead, pathogen surveillance is constant. Previous reciprocal grafting studies using BSR-resistant and -susceptible lines (Bachman and Nickell 1999) demonstrated that the root determines the resistance response observed in the stem and leaves. Our analyses support this hypothesis. Of the 21 genes differentially expressed between genotypes at the $R b s 3$ locus, over half were homologous to RLP expressed in the root, highlighting their importance in the resistant response. These genes were differentially expressed between genotypes but few were differentially expressed in response to treatment. Based on fine mapping (Rincker et al. 2016a) and genome-wide association studies (Rincker et al. 2016b), Rincker et al. (2016a) suggested that all BSR resistance is conferred by a single gene located within a 41-kb interval. Three candidate RLP were identified (Glyma.16G169600, Glyma.16G169700, and Glyma. 16G169900). In our study, none of these genes responded to $P$. gregata infection or showed significantly greater expression in the resistant genotype. We demonstrated that differential expression of candidate RLP was limited to the historic $R b s 3$ locus, outside the region identified by Rincker et al. (2016a). Layering the RLP with historical mapping and expression data demonstrated that different clusters of RLP are associated with the $R b s 1, R b s 2$, and $R b s 3$ loci. We suspect that RNA-Seq expression studies using $R b s l$ or $R b s 2$ genotypes would highlight RLP within those loci. Our findings support multiple previous allelism studies that suggest that $R b s 1, R b s 2$, and Rbs3 are distinct loci (Hanson et al. 1988; Sebastian and Nickell 1985; Willmont and Nickell 1989). The complex arrangement of RLP in the Rbs loci has hindered identification of the causal genes.

Our analyses also focused on characterizing defense responses to $P$. gregata infection. Although plants were inoculated in the stem, we observed the strongest treatment response in resistant leaves, clearly indicating that resistance and expression of defense pathways have spread throughout the plants much earlier than BSR symptom development would indicate. Given that this study was conducted 1 week after $P$. gregata infection and little overlap was detected between DEG of the susceptible and resistant genotype, the genes we have identified can serve as markers in BSR resistance screening protocols. Molecular markers for the candidate resistance genes will be developed and validated, allowing genotyping to occur 4 weeks sooner than phenotyping allows. Molecular screening would be faster and more efficient than current phenotyping methods for BSR. Our results demonstrate that regulation of genes associated with defense, DNA replication, and iron homeostasis are the hallmarks of resistance to $\mathrm{P}$. gregata.

In conclusion, we report the first expression analyses of $P$. gregata infection of soybean. We find that infection results in the differential expression of genes relating to defense, DNA replication, and iron homeostasis. In addition, 12 candidate genes in the $R b s 3$ locus are associated with $R$-gene-mediated defense and that gene expression is strongest in roots. These findings present new avenues for future soybean- $P$. gregata research and new methodical approaches that could have broad implications for other diseases and abiotic stressors affecting soybean and other crops. Further, our work has identified potential novel SNP sequences that will be used for markerassisted selection, fine mapping, and characterizing novel BSR resistance sources. 


\section{MATERIALS AND METHODS}

Experimental design and inoculation.

Two soybean lines were used: resistant line PI 437970 (Hanson et al. 1988) containing $P$. gregata resistance gene $R b s 3$, and susceptible line Corsoy 79 (Bernard and Cremeens 1988). From each genotype, 40 seeds were planted into separate 3.8-by-21-cm cone-tainers (Stuewe and Sons, Corvallis, OR) with Metro-Mix 900 potting soil (Sun Grow Horticulture, Agawam, MA) in a growth chamber arranged in a completely randomized design. Temperature was set to a constant $19 \pm$ $1.5^{\circ} \mathrm{C}$ with a $16-\mathrm{h}$ photoperiod, provided by fluorescent and incandescent light bulbs. Growth chambers were furnished with three types of bulbs: 36 bulbs at $215 \mathrm{~W}$, each producing 10,125 lumens of light; 6 bulbs at $115 \mathrm{~W}$, each producing 4,030 lumens of light; and 30 bulbs at $52 \mathrm{~W}$, each producing 610 lumens of light. Pots were watered daily until saturation and fertilized weekly with a 24-8-16 fertilizer mixture.

The $P$. gregata spore suspension was prepared using isolate $\mathrm{Oh}_{2-3}$ (Lewers et al. 1999), a single-spore isolate of $\mathrm{Oh}_{2}$ (Eathington et al. 1995) originally provided by Cecil Nickell at the University of Illinois. Cultures were grown on green bean extract medium and incubated for 60 days at room temperature with ambient light, as described by McCabe et al. (2016). $P$. gregata spores were suspended in a $1.2 \%$ water agar paste and adjusted to a final spore concentration of $2.7 \times 10^{7}$ spores/ml using a hemocytometer. Two weeks after planting, 20 plants from each genotype were inoculated with $P$. gregata by injecting the soybean stem with approximately $20 \mu \mathrm{l}$ of the suspension $2 \mathrm{~cm}$ above the soil line using an 18-gauge needle. Simultaneously, 20 plants from each genotype were similarly inoculated with $20 \mu \mathrm{l}$ of a spore-free suspension consisting solely of $1.2 \%$ water agar, serving as the mock-inoculated control.

\section{Tissue collection.}

Samples were collected 1, 2, 3, and 4 weeks after inoculation. At each time point, leaf, stem, and root tissues were collected from three plants in each of the four treatments: resistantinfected, resistant-mock, susceptible-infected, and susceptiblemock. The first fully expanded trifoliate leaf, the stem section between the cotyledon and the unifoliate, and the whole root were collected. Samples were flash frozen in liquid nitrogen and stored at $-80^{\circ} \mathrm{C}$.

\section{Disease severity assays.}

Eight plants of each genotype (Corsoy 79 and PI 437970)treatment (infected and mock) combination were phenotyped 5 weeks after infection to verify expected BSR symptoms. BSR severity was assessed using three measurements: foliar severity, stem severity, and stem recovery of $P$. gregata, as described by McCabe et al. (2016). BSR foliar severity was evaluated on each plant using a scale of 1 to 7, with 1 representing the most severe symptoms. Stem severity was defined as the length of brown tissue discoloration in the pith or vascular tissue compared with total plant height. To measure $P$. gregata recovery, plants were sterilized and five evenly spaced, 1-cm-long stem segments were cut from the plant, plated in sequential order on green bean extract agar, and allowed to grow in the dark at room temperature. After 2 weeks, the stem segments on each plate were evaluated for the presence of $P$. gregata colonies and evaluated using a scale of 1 to 5, with 5 representing most severe infection (McCabe et al. 2016; Perez et al. 2010; Tabor et al. 2003).

\section{RNA isolation and RNA-Seq.}

Flash-frozen tissue was ground with a mortar and pestle in liquid nitrogen. A Qiagen RNeasy Plant Mini Kit (Qiagen, Germantown, MD) was used to extract RNA following the manufacturer's recommended protocol. Contaminating DNA was removed with an Ambion TURBO DNA-free kit (Ambion, Austin, TX). A Qiagen RNeasy MiniElute Cleanup Kit (Qiagen) was used to clean and concentrate the RNA. RNA from samples collected at 2, 3, and 4 weeks postinoculation was degraded and not suitable for RNA-Seq analysis. Therefore, only the 36 tissue samples from 1 week postinoculation were used in this study. RNA quality of the samples was confirmed using an Agilent 2100 Bioanalyzer (Agilent, Santa Clara, CA). RNA was considered acceptable if the RNA integrity number was greater than seven. Library preparation and single-end sequencing of the 1-week-postinoculation samples was conducted with $4 \mu \mathrm{g}$ of total RNA using the Illumina HiSeq 2500 platform (Illumina, San Diego, CA) at the Iowa State University DNA facility. All libraries were prepared simultaneously and all samples were sequenced in the same run. Multiple lanes were used for sequencing; however, each lane contained a complete replication to avoid lane bias. Library preparation was unsuccessful for one susceptible mock-infected root sample.

\section{Bioinformatics and statistical analyses.}

The 100-bp reads from 35 separate libraries were trimmed prior to alignment to remove adaptor sequences (Scythe), sequencing artifacts (FASTX trimmer), and low-quality bases (Sickle). Reads were then aligned to version 2 of the Williams 82 reference genome sequence (Wm82.a2.v1) (Schmutz et al. 2010) using TopHat, version 2.0.3 (Trapnell et al. 2009). Unreliably mapped reads were removed using Samtools ( $\mathrm{Li}$ et al. 2009) and mapping files (bamfiles) were imported into the statistical program R (R CoreTeam 2014) using Rsamtools (Morgan and Pages 2013). The gene feature file corresponding to the reference genome was imported using rtracklayer (Lawrence et al. 2009). The number of reads per sample aligning to each gene were counted using GenomicAlignments (Lawrence et al. 2013). Only genes with $\log 2 \mathrm{cpm}>1$ in at least two replicates were used in the analysis.

Data were normalized using the trimmed mean of $\mathrm{M}$ values (Robinson and Oshlack 2010) in the Bioconductor package edgeR (McCarthy et al. 2012; Robinson and Smyth 2007, 2008; Robinson et al. 2010; Zhou et al. 2014). The R graphics program ggplot2 (Wickham 2009) was used to generate principal component and biological coefficient of variance plots to visually compare sample replicates for technical reproducibility, as described by Yin et al. (2013). In all, 5 samples (1 resistantinfected stem, 1 resistant-mock infected stem, 1 resistantinfected root, 1 resistant-infected leaf, and 1 resistant-mock infected leaf) were removed because visual analyses indicated that these samples were substantially different from the other 12 samples of the same tissue. For each tissue, the remaining 10 samples were analyzed using edgeR to identify significantly DEG (FDR $<0.001$ ), responding to $P$. gregata treatment in both genotypes, genotype-specific responses to $P$. gregata infection, treatment-genotype interactions, and general genotype differences.

\section{Identification of candidate $R$ genes at $\boldsymbol{R} b$ s loci in Williams 82.}

The Williams 82 reference genome was used to identify predicted proteins corresponding to the $R b s$ loci with homology to known $R$ genes. The annotations of 687 predicted proteins located between markers Satt215 and Satt431 (Gm16, 28.9 to $36.2 \mathrm{Mbp}$ ) were analyzed using the SoyBase genome annotation report page. Annotations were screened for PFAM domains PF05560 (LRR domain) and PF00931 (NB-ARC domain) and PANTHER domain PTHR24420 (RLP domain). InterProScan software (Jones et al. 2014) was used to identify additional structural domains. The 65 full-length RLP were aligned with T-Coffee, version 7.54 (Notredame et al. 2000) and protein 
sequences were trimmed to retain only the RLP B-domain (Fritz-Laylin et al. 2005). To overlay the candidate $R$ genes with historical mapping data, we obtained position information for each gene and marker, relative to the Williams 82 genome. Custom R code using ggplot2 (Wickham 2009) allowed for visualization of genes, markers, and expression data.

\section{De novo assembly of the expanded $R$ bs 3 locus.}

$R b s 3$ was mapped between markers K375 and G815 (33.3 to 34.3 Mbp) (Lewers et al. 1999). Fine mapping and genomewide association study approaches by Rincker et al. (2016a) and Rincker et al. (2016b) suggested that $R b s 1, R b s 2$, and $R b s 3$ each map to genomic positions within 32.7 to $33.4 \mathrm{Mbp}$ on chromosome 16 . We combined these regions and conducted a de novo assembly of chromosome 16 from bases $32,725,851$ to 34,250,536. Samtools ( $\mathrm{Li}$ et al. 2009) was used to extract reliably mapped reads from each alignment file (bamfile) used for RNA-Seq. Trinity (version 2.0.6) (Grabherr et al. 2011) was used to develop a de novo assembly of transcripts from this region resulting in 508 transcripts, representing 289 genes. Median transcript length was 713 bases, with an average transcript length of 1,168 bases. The corresponding region in the reference genome contains 140 high-confidence genes (Glyma. 16 G167600 to Glyma.16G181500) encoding 220 unique predicted transcripts. To assign function, sequences were compared with predicted soybean transcripts in version Williams82.a2.v1 using BLASTN $\left(\mathrm{E}<10^{-50}\right)$ (Altschul et al. 1997) and to predicted proteins in Arabidopsis (TAIR version 10) using BLASTX $\left(\mathrm{E}<10^{-6}\right)$. Only the top BLAST hits are reported. To identify significantly differentially expressed transcripts in our de novo assembly, reads used to create the assembly were mapped back to the de novo assembly using Bowtie (version 2) (Langmead et al. 2009). RNA-Seq by Expectation-Maximization software (Li and Dewey 2011) was used to develop count matrices for all transcripts in each tissue. Each tissue count matrix was imported into R (R CoreTeam 2014) to identify differentially expressed transcripts in the expanded $R b s 3$ locus. The differentially expressed pipeline described above was used to identify DEG.

\section{SNP identification at $R b s$ loci.}

Our original mapping files (bamfiles) from all genotypes, treatments, and tissues were sorted and merged to the Williams 82 reference genome using samtools (Li et al. 2009). Variant calling between the resistant and susceptible genotypes at the expanded $R b s 3$ region (Gm16, 32,725,851 to 34,250,526) was performed using the programs samtools mpileup (Li et al. 2009) and bcftools (Li 2011). The resulting variant call format file was sorted manually and SNP with a quality score less than 25 were removed.

\section{Hierarchical clustering heat maps.}

In order to identify clusters of DEG responding similarly to $P$. gregata infection across treatments, genotypes, and tissues, we performed hierarchical clustering analysis based on expression profiles of DEG for treatment, treatment-genotype interaction, and genotype responses across tissues. Hierarchical clustering was conducted with gene $\log 2 \mathrm{cpm}$ for each sample using the hclust command in $\mathrm{R}$ (R CoreTeam 2014). Clustering was based on $\mathrm{z}$ scores, measuring the number of standard deviations away a sample is relative to the mean for all samples for a given gene.

\section{Annotation and analysis of DEG.}

DEG were annotated using the SoyBase Genome Annotation Report page. Significantly $(P$ value $<0.05)$ overrepresented biological process GO terms were identified using a Fisher's exact test (Fisher 1960) and Bonferroni correction (Bonferroni
1935) to compare significantly overrepresented GO terms within a cluster to their genome representation. To reduce the number of significantly overrepresented GO terms and eliminate redundancy, any GO terms whose genes completely overlapped were mapped to the largest significantly overrepresented GO term. TF were identified using the SoyDB TF database (Wang et al. 2010). The gene identifiers of TF present in the database were updated to reference the new genome assembly and annotation (Wm82.a2.v1) (Schmutz et al. 2010).

\section{ACKNOWLEDGMENTS}

We thank A. M. Lauter for assistance with RNA extractions and D. Cook for statistical suggestions.

\section{LITERATURE CITED}

Allington, W. B., and Chamberlain, D. W. 1948. Brown stem rot of soybean. Phytopathology 38:793-802.

Altschul, S. F., Madden, T. L., Schäffer, A. A., Zhang, J., Zhang, Z., Miller, W. and Lipman, D. J. 1997. Gapped BLAST and PSI-BLAST: A new generation of protein database search programs. Nucleic Acids Res. 25:3389-3402.

Anderson, J. E., Kantar, M. B., Kono, T. Y., Fu, F., Stec, A. O., Song, Q., Cregan, P. B., Specht, J. E., Diers, B. W., Cannon, S. B., McHale, L. K., and Stupar, R. M. 2014. A roadmap for functional structural variants in the soybean genome. G3: Genes Genomes Genet. 4:1307-1318.

Bachman, M. S., and Nickell, C. D. 1999. Use of reciprocal grafting to study brown stem rot resistance in soybean. Phytopathology 89:59-63.

Bachman, M. S., and Nickell, C. D. 2000. High frequency of brown stem rot resistance in soybean germplasm from central and southern China. Plant Dis. 84:694-699.

Bachman, M. S., Tamulonis, J. P., Nickell, C. D., and Bent, A. F. 2001 Molecular markers linked to brown stem rot resistance genes, Rbs 1 and $R b s 2$, in soybean. Crop Sci. 41:527-535.

Berardini, T. Z., Mundodi, S., Reiser, L., Huala, E., Garcia-Hernandez, M., Zhang, P., Mueller, L. A., Yoon, J., Doyle, A., Lander, G., Moseyko, N., Yoo, D., Xu, I., Zoeckler, B., Montoya, M., Miller, N., Weems, D., and Rhee, S. Y. 2004. Functional annotation of the Arabidopsis genome using controlled vocabularies. Plant Physiol. 135:745-755.

Bernard, R. L., and Cremeens, C. R. 1988. Registration of 'Corsoy 79' soybean. Crop Sci. 28:1027.

Bilgin, D. D., Zavala, J. A., Zhu, J., Clough, S. J., Ort, D. R., and DeLucia, E. H. 2010. Biotic stress globally downregulates photosynthesis genes. Plant Cell Environ. 33:1597-1613.

Bolton, M. D. 2009. Primary metabolism and plant defense-fuel for the fire Mol. Plant-Microbe Interact. 22:487-497.

Bonferroni, C. E. 1935. Il calcolo delle assicurazioni su gruppi di teste. Tipografia del Senato. Studi in onore del professore salvatore ortu carboni.

Cheong, Y. H., Chang, H.-S., Gupta, R., Wang, X., Zhu, T., and Luan, S. 2002. Transcriptional profiling reveals novel interactions between wounding, pathogen, abiotic stress, and hormonal responses in Arabidopsis. Plant Physiol. 129:661-677

Danzer, J., Mellott, E., Bui, A. Q., Le, B. H., Martin, P., Hashimoto, M., PerezLesher, J., Chen, M., Pelletier, J. M., Somers, D. A., Goldberg, R. B., and Harada, J. J. 2015. Down-regulating the expression of 53 soybean transcription factor genes uncovers a role for SPEECHLESS in initiating stomatal cell lineages during embryo development. Plant Physiol. 168:1025-1035.

Ding, Z., Li, S., An, X., Liu, X., Qin, H., and Wang, D. 2009. Transgenic expression of $M Y B 15$ confers enhanced sensitivity to abscisic acid and improved drought tolerance in Arabidopsis thaliana. J. Genet. Genomics 36:17-29

Eathington, S. R., Nickell, C. D., and Gray, L. E. 1995. Inheritance of brown stem rot resistance in soybean cultivar BSR 101. J. Hered. 86:55-60.

Fisher, S. R. A. 1960. The Design of Experiments. Oliver and Boyd, Edinburgh, United Kingdom.

Fowler, S., and Thomashow, M. F. 2002. Arabidopsis transcriptome profiling indicates that multiple regulatory pathways are activated during cold acclimation in addition to the CBF cold response pathway. Plant Cell 14:1675-1690.

Fritz-Laylin, L. K., Krishnamurthy, N., Tör, M., Sjölander, K. V., and Jones, J. D. 2005. Phylogenomic analysis of the receptor-like proteins of rice and Arabidopsis. Plant Physiol. 138:611-623.

Gao, Q.-M., Venugopal, S., Navarre, D., and Kachroo, A. 2011. Low oleic acid-derived repression of jasmonic acid-inducible defense responses requires the WRKY50 and WRKY51 proteins. Plant Physiol. 155:464-476. 
Gendrel, A.-V., Lippman, Z., Yordan, C., Colot, V., and Martienssen, R. A. 2002. Dependence of heterochromatic histone H3 methylation patterns on the Arabidopsis gene DDM1. Science 297:1871-1873.

Gollhofer, J., Schläwicke, C., Jungnick, N., Schmidt, W., and Buckhout, T. J. 2011. Members of a small family of nodulin-like genes are regulated under iron deficiency in roots of Arabidopsis thaliana. Plant Physiol. Biochem. 49:557-564.

Grabherr, M. G., Haas, B. J., Yassour, M., Levin, J. Z., Thompson, D. A., Amit, I., Adiconis, X., Fan, L., Raychowdhury, R., Zeng, Q., Chen, Z., Mauceli, E., Hacohen, N., Gnirke, A., Rhind, N., di Palma, F., Birren, B. W., Nusbaum, C., Lindblad-Toh, K., Friedman, N., and Regev, A. 2011. Full-length transcriptome assembly from RNA-Seq data without a reference genome. Nat. Biotechnol. 29:644-652.

Gray, L. E. 1972. Effect of Cephalosporium gregatum on soybean yield. Plant Dis. Rep. 56:580-581.

Gutterson, N., and Reuber, T. L. 2004. Regulation of disease resistance pathways by AP2/ERF transcription factors. Curr. Opin. Plant Biol. 7:465-471.

Hanson, P. M., Nickell, C. D., Gray, L. E., and Sebastian, S. A. 1988 Identification of two dominant genes conditioning brown stem rot resistance in soybean. Crop Sci. 28:41-43.

Harrington, T. C., and McNew, D. L. 2003. Phylogenetic analysis places the Phialophora-like anamorph genus Cadophora in the Helotiales. Mycotaxon 87:141-152.

Harrington, T. C., Steimel, J., Workneh, F., and Yang, X. B. 2003. Characterization and distribution of two races of Phialophora gregata in the north-central United States. Phytopathology 93:901-912.

Jones, P., Binns, D., Chang, H.-Y., Fraser, M., Li, W., McAnulla, C., McWilliam, H., Maslen, J., Mitchell, A., Nuka, G., Pesseat, S., Quinn, A. F., Sangrador-Vegas, A., Scheremetjew, M., Yong, S. Y., Lopez, R., and Hunter, S. 2014. InterProScan 5: Genome-scale protein function classification. Bioinformatics 30:1236-1240.

Kangasjärvi, S., Neukermans, J., Li, S., Aro, E.-M., and Noctor, G. 2012. Photosynthesis, photorespiration, and light signalling in defence responses. J. Exp. Bot. 63:1619-1636.

Klos, K. L. E., Paz, M. M., Fredrick Marek, L., Cregan, P. B., and Shoemaker, R. C. 2000. Molecular markers useful for detecting resistance to brown stem rot in soybean. Crop Sci. 40:1445-1452.

Koenning, S., and Wrather, J. A. 2015. Estimates of Soybean Yield Reductions Caused by Diseases in the United States. University of Illinois at UrbanaChampaign Crop Science Extension and Outreach, Urbana, IL, U.S.A.

Kruijt, M., de Kock, M. J., and de Wit, P. J. 2005. Receptor-like proteins involved in plant disease resistance. Mol. Plant Pathol. 6:85-97.

Langmead, B., Trapnell, C., Pop, M., and Salzberg, S. L. 2009. Ultrafast and memory-efficient alignment of short DNA sequences to the human genome. Genome Biol. 10:R25.

Lawrence, M., Gentleman, R., and Carey, V. 2009. rtracklayer: An R package for interfacing with genome browsers. Bioinformatics 25:1841-1842.

Lawrence, M., Huber, W., Pagès, H., Aboyoun, P., Carlson, M., Gentleman, R., Morgan, M. T., and Carey, V. J. 2013. Software for computing and annotating genomic ranges. PLOS Comput. Biol. 9:e1003118.

Lewers, K. S., Crane, E. H., Bronson, C. R., Schupp, J. M., Keim, P., and Shoemaker, R. C. 1999. Detection of linked QTL for soybean brown stem rot resistance in 'BSR 101' as expressed in a growth chamber environment. Mol. Breed. 5:33-42.

Li, B., and Dewey, C. N. 2011. RSEM: Accurate transcript quantification from RNA-Seq data with or without a reference genome. BMC Bioinf. 12:323.

Li, G., Liu, S., Wang, J., He, J., Huang, H., Zhang, Y., and Xu, L. 2014. ISWI proteins participate in the genome-wide nucleosome distribution in Arabidopsis. Plant J. 78:706-714.

Li, H. 2011. A statistical framework for SNP calling, mutation discovery, association mapping and population genetical parameter estimation from sequencing data. Bioinformatics 27:2987-2993.

Li, H., Handsaker, B., Wysoker, A., Fennell, T., Ruan, J., Homer, N., Marth, G., Abecasis, G., and Durbin, R.; 1000 Genome Project Data Processing Subgroup. 2009. The sequence alignment/map format and SAMtools. Bioinformatics 25:2078-2079.

Li, Y., Baldauf, S., Lim, E.-K., and Bowles, D. J. 2001. Phylogenetic analysis of the UDP-glycosyltransferase multigene family of Arabidopsis thaliana. J. Biol. Chem. 276:4338-4343.

Liang, G., Zhang, H., Li, X., Ai, Q., and Yu, D. 2017. bHLH transcription factor bHLH115 regulates iron homeostasis in Arabidopsis thaliana. J. Exp. Bot. 68:1743-1755.

McCabe, C. E., Singh, A. K., Leandro, L. F., Cianzio, S. C., and Graham, M. A. 2016. Identifying new sources of resistance to brown stem rot in soybean. Crop Sci. 56:2287-2296.

McCarthy, D. J., Chen, Y., and Smyth, G. K. 2012. Differential expression analysis of multifactor RNA-Seq experiments with respect to biological variation. Nucleic Acids Res. 40:4288-4297.
McHale, L. K., Haun, W. J., Xu, W. W., Bhaskar, P. B., Anderson, J. E., Hyten, D. L., Gerhardt, D. J., Jeddeloh, J. A., and Stupar, R. M. 2012 Structural variants in the soybean genome localize to clusters of biotic stress-response genes. Plant Physiol. 159:1295-1308.

Morgan, M., and Pages, H. 2013. Rsamtools: Binary alignment (BAM), variant call (BCF), or tabix file import (R package version 1.12.4). Online publication. https://rdrr.io/bioc/Rsamtools/

Notredame, C., Higgins, D. G., and Heringa, J. 2000. T-Coffee: A novel method for fast and accurate multiple sequence alignment. J. Mol. Biol. 302:205-217.

Perez, P. T., Diers, B. W., Lundeen, P., Tabor, G. M., and Cianzio, S. R. 2010. Genetic analysis of new sources of soybean resistance to brown stem rot. Crop Sci. 50:2431-2439.

Plett, J. M., Tisserant, E., Brun, A., Morin, E., Grigoriev, I. V., Kuo, A., Martin, F., and Kohler, A. 2015. The mutualist Laccaria bicolor expresses a core gene regulon during the colonization of diverse host plants and a variable regulon to counteract host-specific defenses. Mol. Plant-Microbe Interact. 28:261-273.

Poppenberger, B., Berthiller, F., Lucyshyn, D., Sieberer, T., Schuhmacher, R., Krska, R., Kuchler, K., Glössl, J., Luschnig, C., and Adam, G. 2003 Detoxification of the Fusarium mycotoxin deoxynivalenol by a UDPglucosyltransferase from Arabidopsis thaliana. J. Biol. Chem. 278 47905-47914.

R CoreTeam. 2014. R: A Language and Environment for Statistical Computing. R Foundation for Statistical Computing, Vienna.

Rincker, K., Hartman, G. L., and Diers, B. W. 2016a. Fine mapping of resistance genes from five brown stem rot resistance sources in soybean. Plant Genome 9: No. 1.

Rincker, K., Lipka, A. E., and Diers, B. W. 2016b. Genome-wide association study of brown stem rot resistance in soybean across multiple populations. Plant Genome 9: No. 2.

Robert-Seilaniantz, A., Grant, M., and Jones, J. D. 2011. Hormone crosstalk in plant disease and defense: More than just jasmonate-salicylate antagonism. Annu. Rev. Phytopathol. 49:317-343.

Robinson, M. D., McCarthy, D. J., and Smyth, G. K. 2010. edgeR: A Bioconductor package for differential expression analysis of digital gene expression data. Bioinformatics 26:139-140.

Robinson, M. D., and Oshlack, A. 2010. A scaling normalization method for differential expression analysis of RNA-seq data. Genome Biol. 11:R25.

Robinson, M. D., and Smyth, G. K. 2007. Moderated statistical tests for assessing differences in tag abundance. Bioinformatics 23:2881-2887.

Robinson, M. D., and Smyth, G. K. 2008. Small-sample estimation of negative binomial dispersion, with applications to SAGE data. Biostatistics 9:321-332.

Schmutz, J., Cannon, S. B., Schlueter, J., Ma, J., Mitros, T., Nelson, W., Hyten, D. L., Song, Q., Thelen, J. J., Cheng, J., Xu, D., Hellsten, U., May, G. D., Yu, Y., Sakurai, T., Umezawa, T., Bhattacharyya, M. K., Sandhu, D., Valliyodan, B., Lindquist, E., Peto, M., Grant, D., Shu, S., Goodstein, D., Barry, K., Futrell-Griggs, M., Abernathy, B., Du, J., Tian, Z., Zhu, L., Gill, N., Joshi, T., Libault, M., Sethuraman, A., Zhang, X. C., Shinozaki, K., Nguyen, H. T., Wing, R. A., Cregan, P., Specht, J., Grimwood, J., Rokhsar, D., Stacey, G., Shoemaker, R. C., and Jackson, S. A. 2010 Genome sequence of the palaeopolyploid soybean. Nature 463:178-183.

Sebastian, S. A., and Nickell, C. D. 1985. Inheritance of brown stem rot resistance in soybeans. J. Hered. 76:194-198.

Simon, C., Langlois-Meurinne, M., Didierlaurent, L., Chaouch, S., Bellvert, F., Massoud, K., Garmier, M., Thareau, V., Comte, G., Noctor, G., and Saindrenan, P. 2014. The secondary metabolism glycosyltransferases UGT73B3 and UGT73B5 are components of redox status in resistance of Arabidopsis to Pseudomonas syringae pv. tomato. Plant Cell Environ. 37:1114-1129.

SoyStats. 2015. A Reference Guide to Important Soybean Facts and Figures. American Soybean Association, St. Louis.

Suzuki, T., Nakajima, S., Inagaki, S., Hirano-Nakakita, M., Matsuoka, K., Demura, T., Fukuda, H., Morikami, A., and Nakamura, K. 2005 TONSOKU is expressed in $\mathrm{S}$ phase of the cell cycle and its defect delays cell cycle progression in Arabidopsis. Plant Cell Physiol. 46:736-742.

Tabor, G. M., Tylka, G. L., Cianzio, S. C., and Bronson, C. R. 2003. Resistance to Phialophora gregata is expressed in the stems of resistant soybeans. Plant Dis. 87:970-976.

Trapnell, C., Pachter, L., and Salzberg, S. L. 2009. TopHat: Discovering splice junctions with RNA-Seq. Bioinformatics 25:1105-1111.

van Leeuwen, W., Ökrész, L., Bögre, L., and Munnik, T. 2004. Learning the lipid language of plant signalling. Trends Plant Sci. 9:378-384.

Wang, Z., Libault, M., Joshi, T., Valliyodan, B., Nguyen, H. T., Xu, D., Stacey, G., and Cheng, J. 2010. SoyDB: A knowledge database of soybean transcription factors. BMC Plant Biol. 10:14.

Wickham, H. 2009. ggplot2: Elegant Graphics for Data Analysis. Springer Science \& Business Media, New York. 
Willmont, D. B., and Nickell, C. 1989. Genetic analysis of brown stem rot resistance in soybean. Crop Sci. 29:672-674.

Yin, T., Majumder, M., Chowdhury, N. R., Cook, D., Shoemaker, R., and Graham, M. 2013. Visual mining methods for RNA-Seq data: Data structure, dispersion estimation and significance testing. J. Data Min. Genomics Proteomics. doi.org/10.4172/2153-0602.1000139

Zhang, H., Huang, L., Dai, Y., Liu, S., Hong, Y., Tian, L., Huang, L., Cao, Z., Li, D., and Song, F. 2015. Arabidopsis AtERF15 positively regulates immunity against Pseudomonas syringae pv. tomato DC3000 and Botrytis cinerea. Front. Plant Sci. 6:686.

Zhang, Z., Wang, J., Zhang, R., and Huang, R. 2012. The ethylene response factor AtERF98 enhances tolerance to salt through the transcriptional activation of ascorbic acid synthesis in Arabidopsis. Plant J. 71:273-287.
Zhou, X., Lindsay, H., and Robinson, M. D. 2014. Robustly detecting differential expression in RNA sequencing data using observation weights. Nucleic Acids Res. 42:e91.

\section{AUTHOR-RECOMMENDED INTERNET RESOURCES}

National Center for Biotechnology Sequence Read Archive: https://www.ncbi.nlm.nih.gov/sra

Scythe: https://github.com/vsbuffalo/scythe

FASTX trimmer: http://hannonlab.cshl.edu/fastx_toolkit/

Sickle: https://github.com/najoshi/sickle

SoyBase Genome Annotation Report: https://www.soybase.org/genomeannotation/ 